\title{
Modeling and Analysis of Inductive Coil ELF Sensor
}

\author{
Rajendra Aparnathi*, Vedvyas Dwivedi \\ Faculty of Technology and Engineering, C. U. Shah University, Wadhwancity, Surendranagar, Gujarat \\ ${ }^{*}$ Corresponding author, e-mail: rajendraaparnathi@live.com
}

\begin{abstract}
Mathematical statistical analysis of inductive loop coil-sensor is carried out for its magnetic field effects operating on extremely low frequency $(<30 \mathrm{~Hz})$. A system using resister, inductor, and capacitor effects finds resonance frequency for this loop sensor and its sensitivity as ferromagnetic effect. The design methods for these coils with air and ferromagnetic cores are technically compared and summarized, which are also known and used as search coils or pickup coils or magnetic loop coil sensors. The amplitude and bandwidth of the frequency components are compared to the standardized normal spectrum. This paper also presents the applications of coil sensor as magnetic coil.
\end{abstract}

Keywords: coil sensor, magnetic loop coil, ferromagnetic core, extreme low frequency.

Copyright $(2015$ Institute of Advanced Engineering and Science. All rights reserved.

\section{Introduction}

Induction sensor is also known as search coil because their measuring principles are based on varying magnetic field measurement. The knowledge of physical phenomena related to induction magnetometers (induction, magnetic, and extremely low noise amplification) constitutes of strong background to address design of other types of magnetometer and their applications [1]. The induction sensor principle helps derive directly from Faraday's law equation (1) [1].

$$
e=\frac{d \phi}{d t}
$$

Where, $\emptyset=\iint(s) \overrightarrow{B d s}$ is magnetic flux through a coil over a surface (s) the voltage is proportional to the time derivative of the flux, thus by principle, dc magnetic field cannot be measure with a static coil, higher will be the frequency higher will be the output voltage (n) coil of section (s) into an homogenous induction magnetic field (B) equation become (2).

$$
e=n S \frac{d B}{d t}
$$

This signal $\mathrm{n}$ turns coils, is design as air coil induction sensor. As increase in sensitivity of air coil increasing number of turns ( $n$ ) or air coil surface $(S)$. In applications where the size, mass and performance of the sensor are not too stringent the air-coil induction sensor is an efficient way to get magnetic field variation are two other advantages of the air coil induction sensor [2-3].

Important ways to improve the sensitivity of an induction sensor consist in using a ferromagnetic core. In that configuration, in that configuration, the ferromagnetic core acts as a magnetic amplifier and coil is wounded around the ferromagnetic core inter-digital electrodes are among the most commonly used periodic electrode structures. Recent advances in such fields as nondestructive testing (NDT), micro -electromechanical systems (MEMS), telecommunications, chemical sensing, piezoacoustics, and biotechnology involve -nterdigital electrodes in very different ways. At the same time, a number of common features are shared among these applications. The purpose of this paper is to outline common features and to highlight the differences of sensor geometry, manufacturing techniques, choice of materials, 
analytical and numerical modeling, design optimization, system integration, and data analysis. It is difficult and perhaps even excessive to maintain equally deep and comprehensive treatment of all these subjects. Instead, the fringing electric field sensors are given the deepest emphasis in this manuscript [4]. Significant aspects of other types of sensors are discussed, while repetition is avoided. References are provided to major review papers and books in each section devoted to a particular field of inter digital electrode applications, such as dielectric imaging, acoustic sensors, and MEMS $[1,5]$.

One way to classify the various magnetic sensors is by the field sensing range. The sensor can be arbitrarily divided into three categories low field, medium field and high field sensing. The technology for sensing magnetic fields has also evolved driven by the need for improved sensitivity, smaller size, and compatibility with electronic systems mention Figure 1.

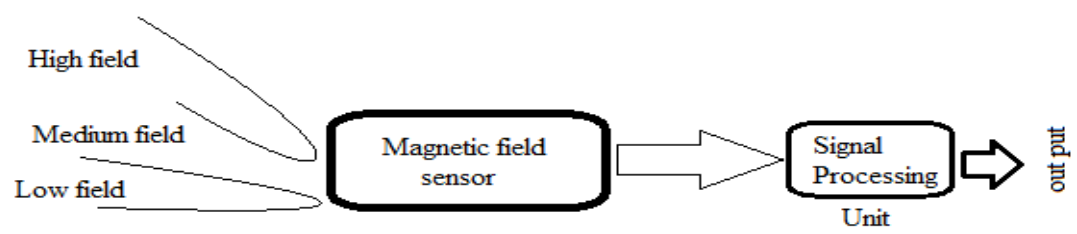

Figure 1. Filed Sense Magnetic Field sensor

A typical single-coil variable-reluctance noise sensor is illustrated in Figure 1. The sensor consists of three elements: a ferromagnetic core sensor in the shape of a semicircular ring, a variable air gap, and a ferromagnetic plate. The total reluctance of the magnetic circuit is the sum of the individual reluctances: in this research paper presented Design inductive coil parameter and statical analysis and preamplifier parameters [6].

\section{Research Method}

\subsection{Design Inductive Sensor Coil Parameter}

The main goal of this review is to summarize the existing knowledge about induction coil sensors, including old, often forgotten publications as well as new developments. Firstly, two main designs of coil sensor (with air cores and ferromagnetic cores) are described. Then, their frequency response is analysed taking into account the type of sensor and their associated output electronics. Secondly, particular induction sensors are discussed and this is followed by a description of the most common application of this type of transducer: magnetic antennae. The relatively low sensitivity of an air coil sensor and problems with its miniaturization can be partially overcome by the incorporation of a ferromagnetic core, which acts as a flux concentrator inside the coil. For a coil with a ferromagnetic core, Equation (1) can be rewritten as Modern soft magnetic materials exhibit a relative permeability, $\mu$, larger than 105 , so this can result in a significant increase of the sensor sensitivity. However, it should be taken into account that the resultant permeability of the core, $\mu \mathrm{c}$, can be much lower than the material permeability [6]. This is due to the demagnetizing field effect defined by the demagnetizing factor $\mathrm{N}$, which is dependent on the geometry of the core If the permeability $\mu \mathrm{r}$ of a material is relatively large (which is generally the case) the resultant permeability of the core $\mu \mathrm{c}$ depends mainly on the demagnetizing factor $\mathrm{N}$. Thus, in the case of a high permeability material, the sensitivity of the sensor depends mostly on the geometry of the core [7].

The demagnetizing factor $\mathrm{N}$ for an ellip-soidal core depends on the core length $\mathrm{Ic}$ and core diameter Dc according to an appro-ximate Equation (3).

$$
\begin{gathered}
V=-\mu_{o} \cdot \mu_{r} \cdot n \cdot A \frac{d H}{d t} \\
\mu_{c}=\frac{\mu_{r}}{1+N \cdot\left(\mu_{r}-1\right)}
\end{gathered}
$$




$$
N \cong \frac{D_{c}^{2}}{l_{c}^{2}} \cdot\left(\ln \frac{2 l_{c}}{D_{c}}-1\right)
$$

It can be derived from Equation (4) that in order to obtain a small value of $\mathrm{N}$ (and a large resultant permeability $\mu \mathrm{c}$ ) the core should be long and with small diameter. Let us consider the dimensions of a search coil sensor optimized for a large sensitivity as described in [8]. The core was prepared from amorphous ribbon (Metglas 2714AF) with dimensions $\mathrm{lc}=300 \mathrm{~mm}$ and $\mathrm{Dc}=10 \mathrm{~mm}$ (aspect ratio equal to 30). Substituting these values into equation (4) we obtain $\mathrm{N}$ $\sim=3.5 \times 10-3$ which means that the sensitivity is about 300 times larger in comparison with the air-coil sensor. Therefore, the use of a core made of a soft magnetic material leads to a significant improvement of the sensor sensitivity. However, this enhancement is achieved with the sacrifice of one of the most important advantages of the air coil sensor the linearity. The core, even if made from the best ferromagnetic material, introduces to the transfer function of the sensor some nonlinear factors which depend on temperature, frequency, flux density, etc. Additional magnetic noise [8] also decreases the resolution of the sensor. Moreover, the ferromagnetic core alters the distribution of the investigated magnetic field (or flux density), which can have important consequences. High permeability core coil sensor is most of used in high sensitivity or dimension limitation is important. It is typical geometry or air sensor is presented in inductive coil Figure 2 and inductive antenna coil presented in Figure 3.

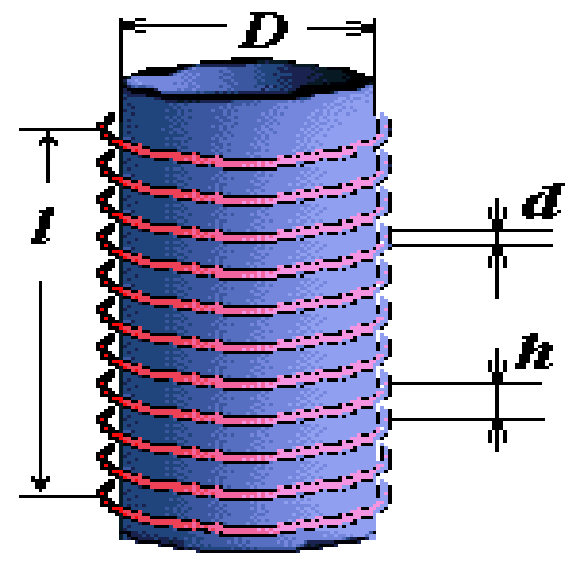

Figure 2. Inductive Coil with Parameter

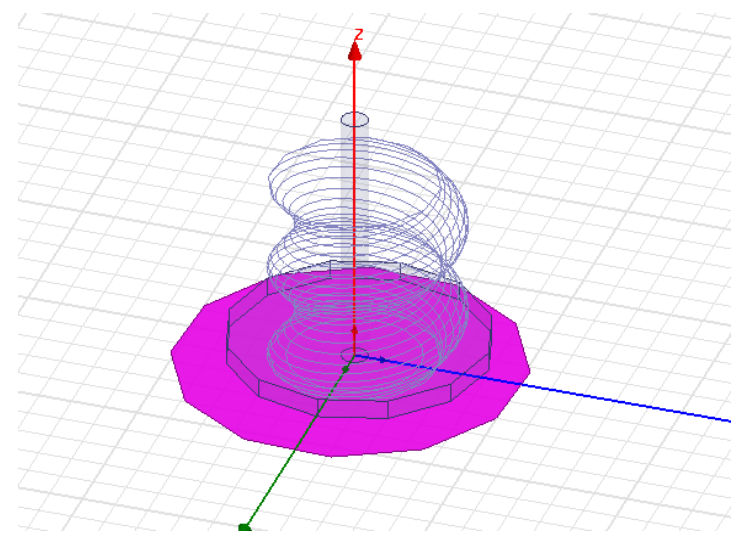

Figure 3. Design Inductive Coil in Simulator

We will start with the description of magnetic amplification provided by a ferromagnetic core. Our description will rely on a simplified modelling of demagnetizing field [9]. Demagnetizing field energy modelling is still of great importance for micro magnetism studies of magnetic sensors. The search coil demagnetizing field effect study has the advantage to be a pedagogic application and to give magnitude scales to the designer through the apparent permeability concept which is at the source of the magnetic amplification of a ferromagnetic core [10]. This last point remains a common denominator of many magnetic sensors. When a magnetic field is applied on a ferromagnetic material, this one becomes magnetized. This magnetization, linked to the magnetic field as expressed, implies an increase of flux density in Equation (5).

$$
\begin{aligned}
& \vec{M}=x \vec{H} \\
& \vec{B}=\mu_{0}(\vec{H}+\vec{M})
\end{aligned}
$$

A way to increase magnetic amplification is to use magnetic concentrators at the ends of the ferromagnetic core (3). 
Let us consider a ferromagnetic core using magnetic concentrators of length ( $L$ ), center diameter (d) and ends diameter (D). High-permeability core coil sensors are often used in the case when high sensitivity or dimension limitations are important. The mention bellow equation is presented apparent permeability.

$$
\mu_{\text {apparent }}=\frac{B_{n}}{B_{e x t}}=\frac{\mu_{r}}{1+N_{z}(L / D) \frac{d^{\prime 2}}{D^{2}}\left(\mu_{r}-1\right)}
$$

For a given set length, diameter and magnetic material, an increase of magnetic concentrators diameter will lead to a significant increase of apparent permeability higher than $50 \%$. Thus the mass of winding of coil and as a consequence the thermal noise due to resistance of the winding. A typical geometry of such a sensor is resented in Figure 5 . The optimal value of core diameter $\mathrm{Di}$ has been determined as $\mathrm{Di}=0.3 \mathrm{D}$ [11]. The coil exhibits a resistance which can be mention Equation (7).

$$
R=\rho N\left(\frac{\frac{d+N+\left(d_{\omega}+1\right)^{2}}{L_{\omega}}}{d_{\omega}^{2}}\right)
$$

Where $\rho$ is the electrical resistivity, $d \omega$ is the wire diameter, $t$ is the thickness of wire insulation, $d$ is the diameter on which coil is wounded and $L \Phi$ is the length of the coil [10].

The coil exhibits a self-inductance which can be expressed 10 in case of an induction sensor using ferromagnetic core and using equation.

$$
L=\lambda \frac{N^{2} \mu_{o} \mu_{\text {apperent }} S}{l}
$$

Where $(S)$ is the ferromagnetic core section, $\mu$ o natural vacuum permeability, $\lambda=$ $(I / / \emptyset) 2 / 5$ is correction factor. The different electrical noise signal potential between each turn of the coil consequence a capacitance because of the store energy between the turns of the coil in mention bellow Equation (9) [11].

$$
C=\frac{\pi \varepsilon_{0} \varepsilon_{r} l_{\omega}}{t\left(n_{l}-1\right)}\left(d+2 n_{l}\left(d_{\omega}+t\right)\right)
$$

Where $\varepsilon \circ \varepsilon r$ are respectively the vacuum permittivity and the relative permittivity of the $\mathrm{nl}$ is the number of layers and other parameter are present and given sensor, the element of the inductive coil sensor electro modeling can be determine in Figure 4.

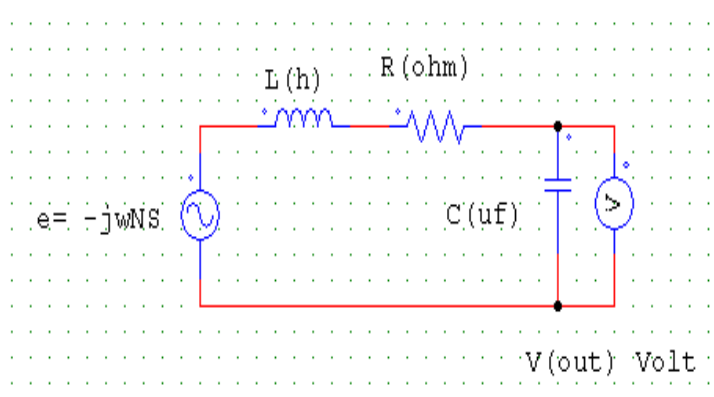

Figure 4. Representation Induction Coil Sensor Fluxmeter 
The transfer function between the output of the inductive coil sensor voltage and flux density can be present in Equation (10) [11].

$$
\frac{V_{\text {out }}}{B}=\left(\frac{-j \omega N S \mu_{\text {apparenter }}}{\left(1-L C \omega^{2}\right)+j R C \omega}\right)
$$

\section{Results and Analysis}

Design Ferromagnetic Core inductive Coil Sensor High permeability ferrite core coil sensors are often used in case when high sensitivity or diameter limitation. The length of the coil $\mathrm{I}$ is recommended to be about $0.7-0.9 \mathrm{lc}$. For such coil dimensions, the output signal $\mathrm{V}$ and SNR ratio at room temperature can be described as It can be concluded from relationships (9) and (10) that in the case of a coil sensor with a ferromagnetic core the most efficient method of improving the sensor performance is to make the length of the core (or rather the ratio l/Di) as large as possible, since the sensitivity is proportional to I3. Figure 5 presents the dependence of the resultant permeability. The choice of the aspect ratio of the core is very important.

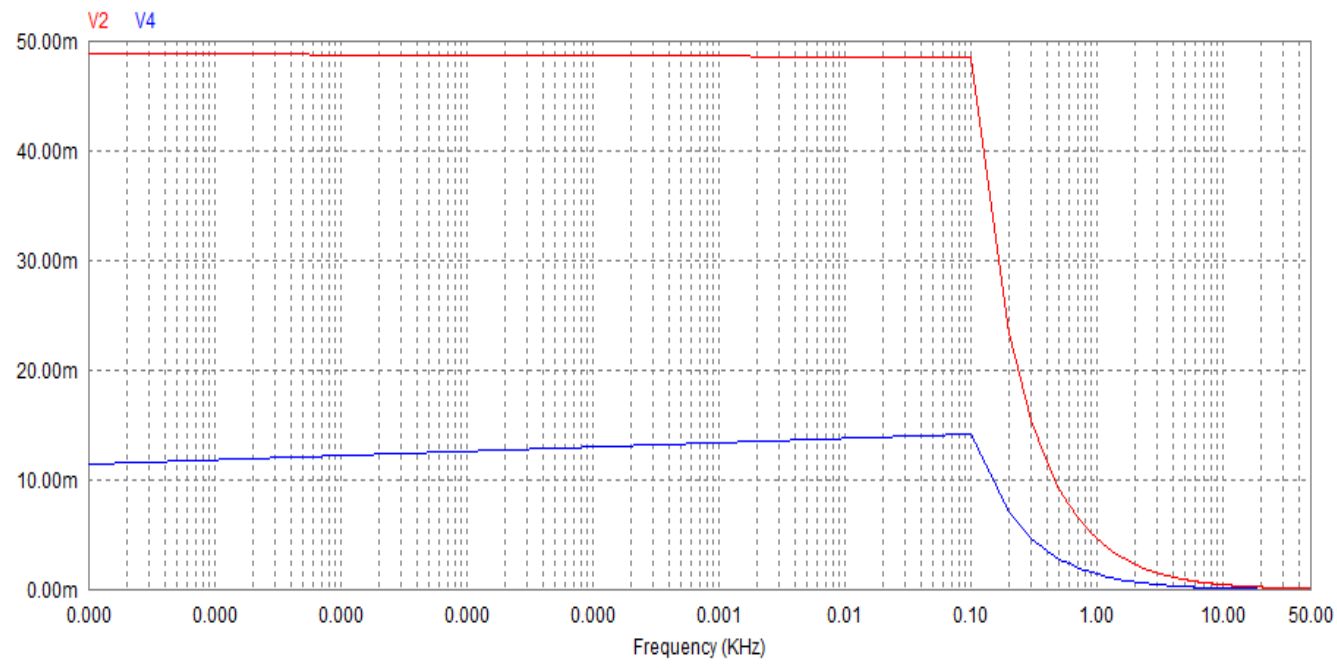

Figure 5 Inductive Coil Output Voltage Respective Frequency

The length should be sufficiently large to benefit from the permeability of the core material. On the other hand, if the aspect ratio is large the resultant permeability depends on the material permeability. This may cause error resulting from the instability of material permeability due to the changes of temperature or applied field frequency. For large values of material permeability the resultant permeability $\mu \mathrm{c}$ practically does not depend on material haracteristics because relation (3) is then:

$$
\mu_{c} \approx \frac{1}{N}
$$

Higher values of material permeability allow the use of longer cores without the risk of the resultant permeability depending on the magnetic characteristic of the material used. As an example, let us consider a low-noise induction magnetometer that is described in [10]. Here, the core was prepared from amorphous ribbon (Metglas 2714AF) with temperature-independent properties and dimensions: length $150 \mathrm{~mm}$, cross-section of the order $5 \times 5 \mathrm{~mm} 2$ (aspect ratio of around 27). A coil of 10000 turns was wound with a $0.15 \mathrm{~mm}$ diameter wire. The noise characteristic of this sensor is presented in Figure 7. The obtained noise level around 0.05 pT $\mathrm{Hz}-1 / 2$ was found to be comparable with the values reported for SQUID sensors. 
The same authors compared the influence of the core material. For an amorphous Metglas core the noise was found to be $0.05 \mathrm{pT} \mathrm{Hz}-1 / 2$, whilst for the same sensor with a permalloy Supermumetal core it exhibited larger noise of $2 \mathrm{pT} \mathrm{Hz}-1 / 2$. Also, a comparison of air coil and ferromagnetic core sensors has been reported [13,14]. Experimental results show that well-designed ferromagnetic core induction sensors exhibited a linearity comparable with aircore sensors.

Sensors with ferromagnetic cores are often used for magnetic investigations in space research [11, 14]. Devices with a core length of $51 \mathrm{~cm}$ and weight of $75 \mathrm{~g}$ (including preamplifier) exhibited a resolution (noise level) of $2 \mathrm{fT} \mathrm{Hz}-1 / 2$ [14]. In an analysis of Earth's magnetic field (OGO search coil experiments) the following three-axis sensors have been used: coil 100000 turns of $0.036 \mathrm{~mm}$ in diameter, core made from nickel-iron alloy $27 \mathrm{~cm}$ long and square $(0.6 \times 0.6 \mathrm{~cm} 2)$ cross-section. Each sensor weighted $150 \mathrm{~g}$ (with half the weight being the core). The sensitivity in this case was $10 \mu \mathrm{V}(\mathrm{nT} \mathrm{Hz})-1$ [10]. The detailed design and optimization of an extremely sensitive three-axis search coil magnetometer for space research is described in [14].

The coil magnetometer developed for the scientific had a noise level of $4 \mathrm{fT} \mathrm{Hz}-1 / 2$ at $30 \mathrm{~Hz}$. Approximation ferrite core effect are present in Table 1.

Table 1. Approximate Maximum Permeabilities

\begin{tabular}{|c|c|c|}
\hline Material & $\mu /(H \mathrm{~m}-1) \mu r$ & Application \\
\hline Ferrite U 60 & 1.00E-05 8 & UHF chokes \\
\hline Ferrite M33 & 9.42E-04 750 & Resonant circuit RM cores \\
\hline Nickel (99\% pure) & $7.54 \mathrm{E}-04600$ & - \\
\hline Ferrite N41 & 3.77E-03 3000 & Power circuits \\
\hline Iron (99.8\% pure) & 6.28E-03 5000 & - \\
\hline Ferrite T38 & 1.26E-02 10000 & Broadband transformers \\
\hline Silicon GO steel & 5.03E-02 40000 & Dynamos, mains transformers \\
\hline supermalloy & 1000000 & Recording heads \\
\hline
\end{tabular}

Table 2. Performance of Inductive Coil Sensor and Parameter

\begin{tabular}{cccccccccccccc}
\hline Sr.No & Rin & Ro & L & C & Zin & Zo & f & B/W & Dc & Ic & D & Di & Dm \\
\hline 1 & 10 & 11.4 & 0.15 & 147 & 10.00009647 & 10.05 & 29.35 & 7.958 & 11 & 330 & 180 & 156.6 & 168.3 \\
2 & 10 & 11.4 & 0.20 & 147 & 10.00014379 & 10.06 & 26.25 & 6.36 & 12 & 360 & 186.2 & 161.994 & 174.097 \\
3 & 10 & 11.4 & 0.25 & 147 & 10.00024177 & 10.05 & 23.96 & 5.3 & 13 & 390 & 175.3 & 152.511 & 163.9055 \\
4 & 10 & 11.4 & 0.30 & 147 & 10.00037641 & 10.04 & 22.18 & 4.541 & 12.5 & 375 & 167.2 & 145.464 & 156.332 \\
5 & 10 & 11.4 & 0.35 & 147 & 10.00023954 & 9.99 & 26.9 & 4.547 & 11.5 & 345 & 168.4 & 146.508 & 157.454 \\
6 & 10 & 11.4 & 0.35 & 100 & 10.00026678 & 10.34 & 21.96 & 4.547 & 9.5 & 285 & 162.5 & 141.375 & 151.9375 \\
7 & 10 & 11.4 & 0.35 & 150 & 10.00014634 & 10.23 & 19.02 & 4.547 & 8.5 & 255 & 156 & 135.72 & 145.86 \\
8 & 10 & 11.4 & 0.35 & 200 & 10.00016365 & 10.25 & 17.01 & 4.547 & 12.8 & 384 & 152.6 & 132.762 & 142.681 \\
9 & 10 & 11.4 & 0.35 & 250 & 10.00009305 & 10.09 & 15.53 & 4.547 & 9.8 & 294 & 153.4 & 133.458 & 143.429 \\
10 & 10 & 11.4 & 0.35 & 300 & 10.00028183 & 10.17 & 14.37 & 4.547 & 11.4 & 342 & 157.3 & 136.851 & 147.0755 \\
11 & 10 & 11.4 & 0.35 & 350 & 10.00007822 & 10.06 & 13.45 & 3.978 & 12.1 & 356.9 & 158.2 & 137.634 & 147.917 \\
12 & 10 & 11.4 & 0.40 & 350 & 10.27141611 & 10.02 & 12.68 & 3.536 & 13.3 & 29.1 & 149.2 & 129.804 & 139.502 \\
13 & 10 & 11.4 & 0.45 & 375 & 10.09063331 & 10.08 & 11.62 & 3.183 & 10.6 & 324 & 161 & 140.07 & 150.535 \\
14 & 10 & 11.4 & 0.50 & 390 & 10.0003692 & 10.08 & 10.86 & 2.89 & 14.2 & 426 & 157 & 136.59 & 146.795 \\
15 & 10 & 11.4 & 0.55 & 390 & 11.72021258 & 11.78 & 9.63 & 2.44 & 9.6 & 288 & 148.6 & 129.282 & 138.941 \\
16 & 6 & 6.7 & 0.98 & 670 & 5.00309007 & 5.12 & 6.2 & 3.824 & 16 & 480 & 153.2 & 133.284 & 143.242 \\
17 & 5 & 6.1 & 0.98 & 670 & 15.00996411 & 15.1 & 6.17 & 2.436 & 14.2 & 426 & 162.3 & 141.201 & 151.7505 \\
18 & 15 & 16.7 & 0.98 & 670 & 10.00008082 & 9.89 & 5.58 & 1.81 & 17.1 & 513 & 148.5 & 129.195 & 138.8475 \\
\hline & & & & & & & & & & & & &
\end{tabular}


To obtain the desired resonance frequency and a resistance noise above the preamplifier voltage noise the diameter of copper wire of $71 \mu \mathrm{m}$ and number of turns of 12200 were selected. The core was built from $170 \mathrm{~mm}$ long $50 \mu \mathrm{m}$ thick annealed FeNiMo 15-80-5 permalloy strips, with a cross section of $4.2 \mathrm{~mm} \times 4.2 \mathrm{~mm}$. The mass of the whole three- axis sensor and the bracket was only $430 \mathrm{~g}$. There are commercially available search coil sensors. The frequency characteristics of this sensor are shown in Figure.6 and the summary of performance and resistance inductance and find all parameter are shown in Table 2 also present in input power Vs output voltage of preamplifier in shown Figure 7 In the Figure 8 is present the value of band weigh, Sensitivity, alpha and beta gain factor vs extreme low frequency 5 to $41.51 \mathrm{~Hz}$ graph relationship. also present ferromagnetic effect in Inductive coil Sensor shown in Figure 9.

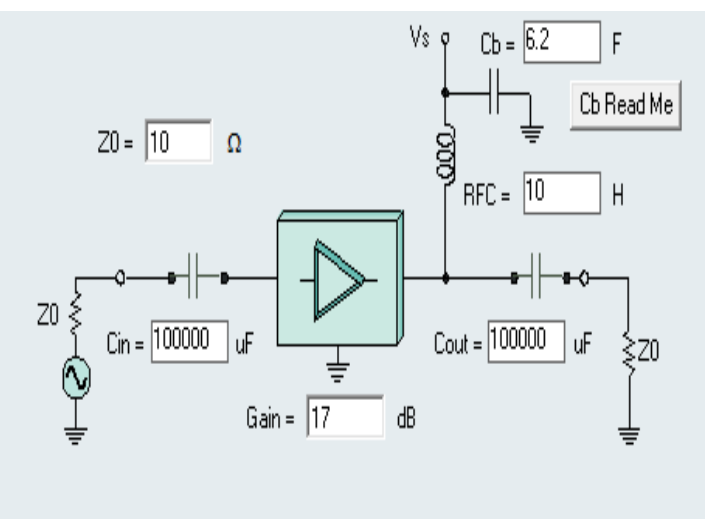

Figure 6. Inductive Coil Sensor Pre-amplifier

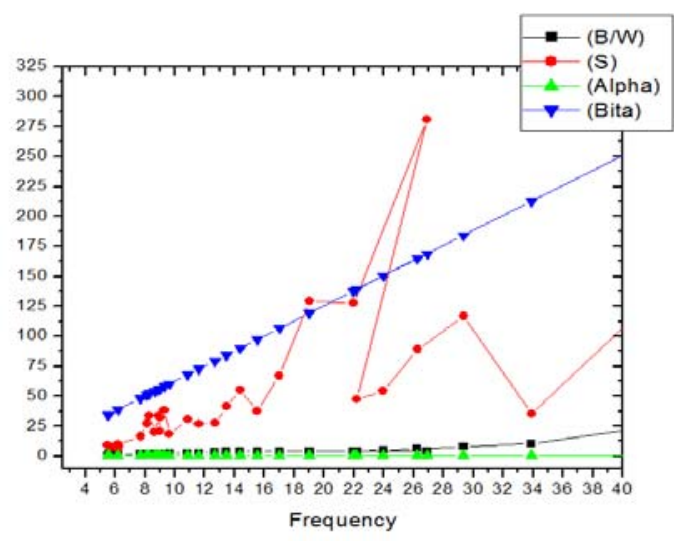

Figure 8. Graph with (B/W, S, Alpha and Betta)

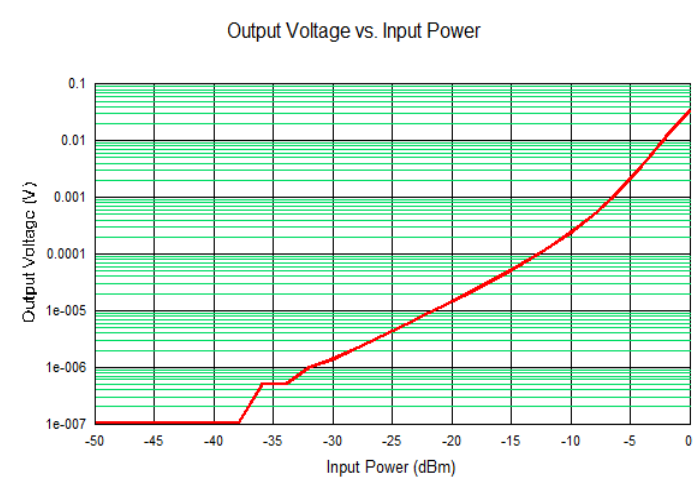

Figure 7. Inductive Coil Sensor Output Voltage vs Input Power Characteristic

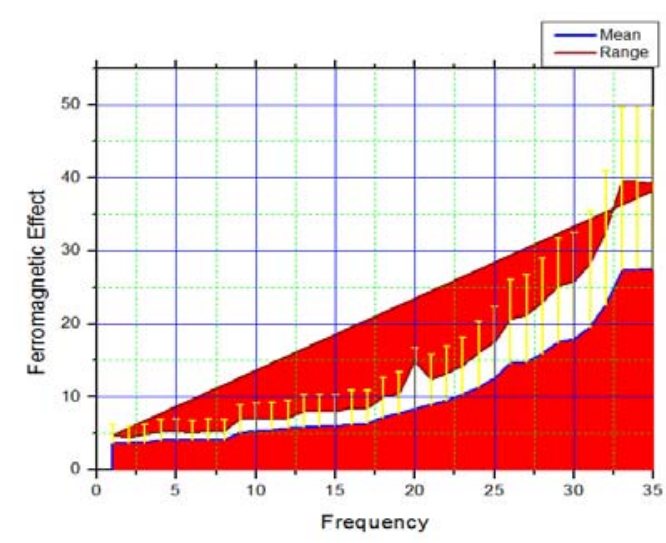

Figure 9. Ferromagnetic Effect for Sensor

\section{Conclusion}

The inductor sensor used for magnetic field measurements have been known extremely low frequency signal or noise. It can be precisely calculation of transfer function and limitation (Boundary) of elf sensor. The induction coil sensor with ferromagnetic core prepared from moden amorphous material exhibit sensitivity. But extremely low frequency for magnetic field applications can be better served by using SQUID methods the most of extremely low frequency sensor, the practical limit of the resolution depended on the possibility of achieving the noise and level of frequency. The designed system allows to enhance the SNR so to improve the minimal resolution of the front-end sensor. Any shape and size of inductive coil sensor that has 
this impedance can be used with the same preamplifier, allowing for a variety of sensitivity and convenience operation without having to make any changes.

\section{References}

[1] Rajendra Aparnathi, Dr Ved Vyas Dwivedi. Design Loop Inductor Coil Sensor for ELF Noise Signals. European Journal of Academic Essays. 2014; 2(1): 21-26.

[2] Rajendra Aparnathi, Dr Ved Vyas Dwivedi. Electro Magnetic Biosensor For Extremely Low Freqency Brain Waves. International Journal of Advanced Research in Electrical, Electronics and Instrumentation Engineering. 2013; 2(9).

[3] S Tumanski. A multi-coil sensor for tangential magnetic field investigations. J. Magn. Magn. Mat. 2002; 242-245: 1153-1156.

[4] H Pfützner, G Krismanic. The needle method for induction tests - sources of error. IEEE Trans. Magn. 2004; 40: 1610-1616.

[5] Alexander V Mamishev. Interdigital Sensor and transducers. Proceedings of The IEEE. 2004; 92(5).

[6] Christophe coillot, Paul Leroy. Induction Megnetometer Principle, Modling and ways of Improvement. LPP Laboratory of Plasma Physics. France. 2010.

[7] Eriksson T, Blomgren J, Winkler D. An HTS SQUID picovoltmeter used as preamplifier for Rogowski coil sensor Physica. 2002.

[8] Yabukami S, Kikuchi K, Yamaguchi M, Arai KI. Magnetic flux sensor principle of microstrip pickup coil. 1997.

[9] Grudzinski E, Rozwalka K. A wideband magnetic field measurements in environment protectionstate of the art and new trends. Przegl. Elektr. 2004; 80: 81-88.

[10] Tumanski S. Thin Film Magnetoresistive Sensors. Bristol: Institute of Physics Publishing. 2000.

[11] Calero C, Piatiini M, Pascual C, Serrano MA. Towards Data Warehouse Quality Metrics. Proceedings of the 3rd Intl. Workshop on Design and Management of Data Warehouses (DMDW). Interlaken. 2009; 39: 2-11.

[12] Yamin L, Wanming C. Implementation of Single Precision Floating Point Square Root on FPGAs. IEEE Symposium on FPGA for Custom Computing Machines. Napa. 2008: 226-232.

[13] Dehmel G. Magnetic field sensors: induction coil (searchcoil) sensors Sensors- A Comprehensive Survey. 1989; 5(6): 205-254.

[14] Ripka P. Induction sensors Magnetic Sensors and Magnetometers. Boston, MA: Artech House. 2001; 47-74.

[15] Mohan N, Undeland TM, Robbins WP. Power Electronics. New York: John Wiley \& Sons. 2005: 1113.

[16] US Patent 44841841984 amorphous antipil ferage. 